2 CARTER, S. F., SZEBESTA, D., DAVEY, S. T., WYATT, R., BRIERLEY, M. C., and FRANCE, P. W.: Amplification at $1.31 \mu \mathrm{m}$ in a $\mathrm{Pr}^{3+}$-doped single-mode fluorozirconate fibre', Electron. Lett., 1991, 27, pp. $628 \quad 629$

3 DURTESTE, Y., MONERIE, M., ALLAIN, J. Y., and POIGNANT, H.: 'Amplification and lasing at $1.3 \mu \mathrm{m}$ in praseodymium-doped fluorozirconate fibres', Electron. Lett., 1991, 27, pp. 626-628

4 ALLAIN, J. Y., MONERIE, M., and POIGNANT, H.: 'Tunable CW lasing around $610,636,695,715,885$, and $910 \mathrm{~nm}$ in praseodymium doped fluorozirconate fibre', Electron. Lett., 1991, 27, pp. 189-190

\section{CACHE VECTOR QUANTISATION ALGORITHM IN VIDEO COMPRESSION}

\section{C.-W. Ku, L.-G. Chen and T.-D. Chiueh}

Indexing terms: Algorithms, Vector quantisation, Video

A new vector quantisation (VQ) algorithm based on a strategy similar to the cache in traditional computers is proposed and simulated. The method can significantly reduce the redundancy in VQ indices. According to the simulated results the bit rate is variable but is much less than that of America, the mean square error is $\sim 23$ and the bit rate is $\sim 0.3 \mathrm{bit} / \mathrm{pixel}$.

Introduction: Recently, vector quantisation (VQ) has been widely investigated for video coding. It is a mapping process for transforming the input vectors to the selected finite vectors called codewords. Because only the indices of these codewords are transmitted or stored, data compression is achieved. The codebook is the core of VQ because it affects the quality of the restored pictures. To maintain the picture quality, a large codebook is usually necessary. Because there is strong spatial and temporal correlation in an image sequence, the indices of the compressed data are concentrated in some local positions, and thus the search time can be significantly reduced. Many algorithms have been proposed to exploit this property, such as address VQ [2], finite state VQ [3], and classified VQ [4] All of these previous algorithms are very complex and some off-line information is needed. The purpose of this Letter is to present a new algorithm for low cost and easy realisation. The method can be applied to image compression or video coding.
Cache vector quantisation algorithm: In computer programs, there are usually arrays, loops, or subroutines. As a result, the access of memory has the property of locality. To exploit this characteristic, a 'cache' is suggested and the data which are expected to be referenced frequently are saved there. Because the access to a cache is faster than the original memory, the execution time is reduced. A similar idea is proposed for VO. The global codebook of cache VQ is generated by the LBG algorithm or others, and the content of the cache is changed to adapt to the input sequence. For each input vector, the best matched codeword in the cache is obtained and the mean square error (MSE) between the input and this codeword is evaluated. If the error is acceptable, the cache's index of this codeword is sent. Otherwise, a full search of the global codebook is applied and the index of the global codebook is transmitted; at the same time, the update procedure for the cache such as first-in-first-out (FIFO), least-frequently-used (LFU) least-recently-used (LRU) etc., is executed. To distinguish between the indices of the cache and global codebook, extra bit overhead is needed. As expected, after a period of time, the codewords in the cache become similar to the input vectors due to the high correlation of video signals in the spatial and temporal domains. Most input vectors therefore 'hit' the codewords in the cache, which is much smaller than the origina codebook. Because the execution time and bit rate for transmitting the codeword's index both depend on the size of the searched set, the average execution time and bit rate are reduced while the picture quality is preserved.

Simulation results: The performance of the full search algorithm is simulated first. Three typical pictures are selected as training data and the codebook is produced by the LBG algorithm. The sequence 'Miss America' is chosen as the outside test set. Those codebooks are represented as global codebooks and the proposed VQ is applied with cache size 16. The MSE of the full search is chosen as the threshold to decide whether there is a 'hit' or not. The comparison between our proposed and full search method is listed in Table 1 . The results are the averages of several frames in the test sequence. Obviously, the improvements of the cache VQ in bit rate and CPU time are significant. The larger the global codebook is, the more benefit is obtained. The effect of cache replacement algorithms and cache size on performance are evaluated by using 1024 codewords. The simulated results are summarised in Table 2 . Fig. 1 shows that a high hit ratio can be easily obtained by using a cache size equal to or greater than 32 words. It is reasonable to assume that as cache size increases, bit rate and hit ratio

Table 1 COMPARISON OF FULL SEARCH VQ AND CACHE VQ

\begin{tabular}{|c|c|c|c|c|c|c|}
\hline \multirow{2}{*}{$\begin{array}{c}\text { Codebook } \\
\text { size }\end{array}$} & \multicolumn{3}{|c|}{ Full search VQ } & \multicolumn{3}{|c|}{ Cache VQ $($ cache size $=16)$} \\
\hline & Bit rate & MSE & CPU time & Bit rate & MSE CPU time & \\
\hline & & & s & & & $\mathbf{s}$ \\
\hline 8 & 0.1875 & $231 \cdot 52$ & $3 \cdot 13$ & - & - & - \\
\hline 16 & 0.2500 & $127 \cdot 71$ & $6 \cdot 32$ & - & - & - \\
\hline 32 & 0.3125 & $52 \cdot 58$ & 11.98 & 0.3280 & $52 \cdot 69$ & 9.93 \\
\hline 64 & $0 \cdot 3750$ & $43 \cdot 46$ & $23 \cdot 33$ & 0.3419 & $43 \cdot 81$ & $16 \cdot 18$ \\
\hline 128 & 0.4375 & $41 \cdot 81$ & $46 \cdot 83$ & 0.3627 & $42 \cdot 04$ & $31 \cdot 32$ \\
\hline 256 & $0 \cdot 5000$ & $30 \cdot 39$ & $94-32$ & 0.3699 & $31 \cdot 57$ & $31 \cdot 35$ \\
\hline 512 & 0.5625 & $24 \cdot 42$ & $186 \cdot 38$ & 0.3862 & $25 \cdot 32$ & $43 \cdot 11$ \\
\hline 1024 & 0.6250 & $21 \cdot 18$ & 373.43 & 0.4038 & $21 \cdot 95$ & $75 \cdot 82$ \\
\hline
\end{tabular}

Test set is 'Miss America'

Table 2 PERFORMANCE OF CACHE VQ ALGORITHM FOR 1024 GLOBAL CODEBOOK

\begin{tabular}{|c|c|c|c|c|c|c|c|c|c|}
\hline \multirow{2}{*}{$\begin{array}{l}\text { Size of } \\
\text { cache }\end{array}$} & \multicolumn{3}{|c|}{ FIFO } & \multicolumn{3}{|c|}{ LFU } & \multicolumn{3}{|c|}{ LRU } \\
\hline & Bit rate & MSE & CPU & Bit rate & MSE & CPU & Bit rate & MSE & CPU \\
\hline 2 & 0.3017 & $22 \cdot 38$ & $92 \cdot 87$ & 0.2951 & $22 \cdot 22$ & $91 \cdot 38$ & 0.3008 & $22 \cdot 37$ & 161.39 \\
\hline 4 & 0.3309 & $22 \cdot 27$ & 87.89 & 0.3207 & $21 \cdot 72$ & $81 \cdot 37$ & 0.3289 & $22 \cdot 24$ & $145 \cdot 53$ \\
\hline 8 & 0.3659 & $22 \cdot 07$ & $82 \cdot 11$ & 0.3557 & $21 \cdot 71$ & $74 \cdot 73$ & 0.3643 & $22-02$ & $136 \cdot 61$ \\
\hline 16 & 0.4038 & 21.95 & 75.82 & 0.3976 & $21 \cdot 50$ & $72 \cdot 94$ & 0.4009 & $21 \cdot 85$ & $127 \cdot 42$ \\
\hline 32 & 0.4416 & 21.89 & $72 \cdot 81$ & 0.4356 & 21.43 & $66 \cdot 95$ & 0.4380 & 21.66 & 118.07 \\
\hline 64 & 0.4873 & $21 \cdot 75$ & 77.95 & 0.4850 & $21 \cdot 38$ & $73 \cdot 53$ & 0.4858 & $21 \cdot 49$ & $129 \cdot 96$ \\
\hline 128 & 0.5361 & $21 \cdot 65$ & $94 \cdot 41$ & 0.5354 & $21 \cdot 37$ & 90.88 & 0.5355 & $21 \cdot 36$ & 160.84 \\
\hline
\end{tabular}

ELECTRONICS LETTERS 5th August 1993 Vol. 29 No. 16 
also increase; however, coding error does not significantly decrease. The CPU time is reduced significantly by cache VQ compared with the full search, as depicted in Fig. 2. When the

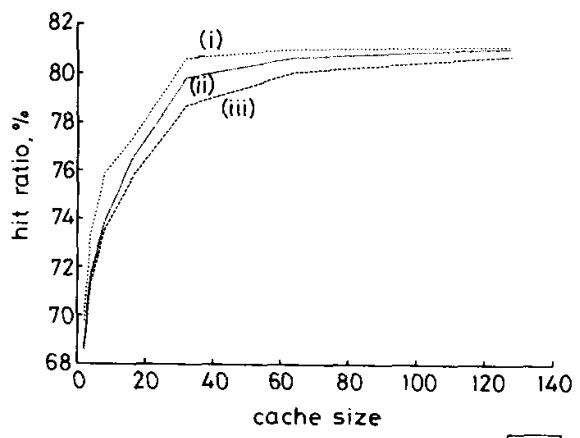

Fig. 1 Effect of cache size on hit ratio

(i) lfu . hit

(ii) Iru . hit

(iii) fifo . hit

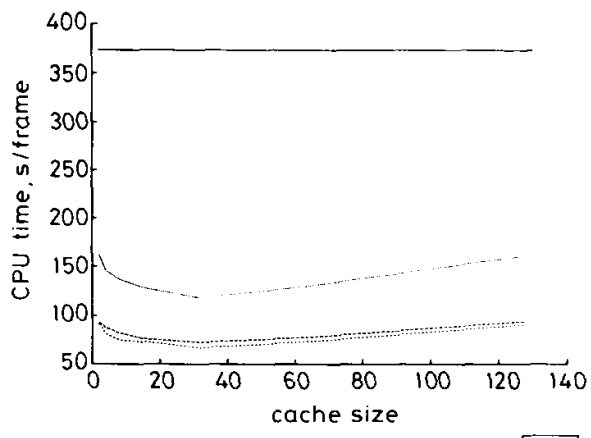

Fig. 2 Comparison of CPU time between cache $V Q$ and full search

- full . cpu

....... Iru . cpu

---- fifo. cp

-..- Ifu. cpu

cache size is 32, the shortest CPU time is obtained. From the simulated results, cache VQ is better than a full search in both bit rate and CPU time and only a small amount of picture quality is sacrificed. The size of cache depends on the applications; if low bit rate is required, a smaller cache size is preferable.

Conclusion: This Letter has described a cache VQ algorithm for video coding. Compared with the traditional full search algorithm, its performance is comparable to a large codebook, but much lower bit rate is required. The CPU time is also reduced because most of the matched codewords can be found in the cache. The cache replacement strategy has little influence on performance. Because of its simplicity, this algorithm is very easy to implement by software or hardware.

(C) IEE 1993

17th June 1993

C.-W. Ku, L.G. Chen and T.-D. Chiueh (Dept of Electrical Engineering, National Taiwan University, Taipei, Taiwan, R.O. China)

\section{References}

1 GERSHO, A., and GRAY, R. M.: 'Vector quantization and signal compression' (Kluwer Academic Publisher, 1991)

2 NASRABADI, $M$ and

vector quantization', IEEE Trans, $1990, C$

CHEN, W.-T., CHANG, R.-F., and WANG, J.-S.: 'Image sequence coding using adaptive finite-state vector quantization', IEEE Trans. CAS for Video Tech., 1992, 2, pp. 15-24

4 RAMAMURTH, B., and GERSHO, A.: 'Classified vector quantization of images', IEEE Trans., 1986, COM-34, pp. 1105-1115

\section{DESIGN OF A CORRUGATED POLARISING} MAIN REFLECTOR

S. S. Chana and C. W. Turner

Indexing terms: Antennas, Antenna reflectors

The realisation of a polarising main reflector for use in a W-band Cassegrain antenna system is described. The main reflector has rectangular grooves cut in its entire surface to change the polarisation from linear to circular. In addition, the use of the FD-TD (finite difference time domain) method for predicting the performance of an axisymmetric dielectric cone subreflector is shown.

Introduction: This Letter addresses the practical realisation of a corrugated polarising main reflector for use in an imaging system. By use of the FD-TD method, mode-matching theory and equivalent circuit method, a design which is relatively simple to manufacture is derived.

The rectangular grooves on the reflector are characterised by analysing infinite flat plate polarisers. Measurements of the performance of the polarising main reflector show a $1.5 \mathrm{~dB}$ axial ratio over a $7 \%$ bandwidth

In addition the application of the FD-TD method for analysing an axisymmetric dielectric cone subreflector is demonstrated. The method will account for the interfaces between the horn aperture and dielectric cone.

Design: A cross-section of a corrugated polarising main reflector for use in a Cassegrain antenna system is shown in Fig. 1.

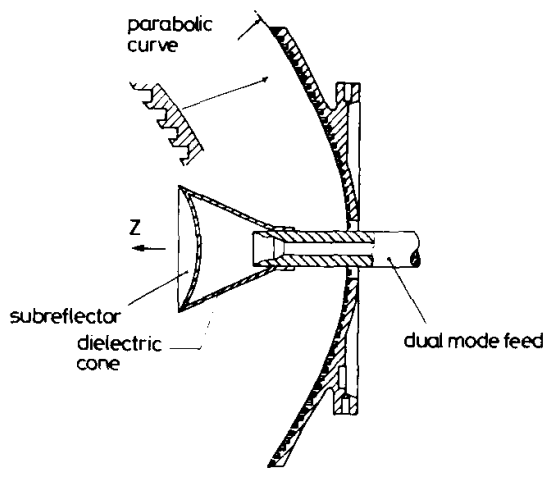

Fig. 1 Corrugated polarising main reflector

A linearly polarised dual mode feed (Potter horn) is used to illuminate a hollow dielectric cone subreflector. The polarisation orientation of the horn is chosen to be $45^{\circ}$ to the groove direction.

To provide mechanical rigidity, polycarbonate $\left(\varepsilon_{p}=3\right)$ is used in the manufacture of the cone, with the wall of the cone approximately half a wavelength thick. A hollow cone is used because of its low weight compared to a solid cone.

To enable the design to be moulded, pressed or machined without having a tool lock problem, the grooves of the polariser are chosen to be parallel to the $z$-axis. As a result the polarising grooves have the shapes shown in Fig. 2 .

A linear polarised wave incident at the surface consists of TE and TM types. If the width $a$ of the groove is chosen to be less than 0.5 wavelength the TE wave will be reflected at XX whereas the TM wave will be coupled into the slot. By choosing $h$, a $90^{\circ}$ phase shift between the two components can be obtained which results in circular polarisation. Only the TE wave is shown in Fig. 2. The TM wave is described by interchanging the $\mathrm{E}$ and $\mathrm{H}$ components of the TE wave.

In the design of the polarising main reflector the slanted grooves are characterised by using FD-TD and the nonslanted grooves by mode-matching theory [2] and the equivalent circuit method [3].

ELECTRONICS LETTERS 5th August 1993 Vol. 29 No. 16 